\title{
Spectral Accuracy and Sulfur Counting Capabilities of the LTQ-FT-ICR and the LTQ-Orbitrap XL for Small Molecule Analysis
}

\author{
Samantha L. Blake, ${ }^{1,2}$ S. Hunter Walker, ${ }^{1}$ David C. Muddiman, ${ }^{1}$ David Hinks, ${ }^{2}$ \\ Keith R. Beck ${ }^{2}$ \\ ${ }^{1}$ W. M. Keck FT-ICR Mass Spectrometry Laboratory, Department of Chemistry, North Carolina State University, Raleigh, \\ NC 27695, USA \\ ${ }^{2}$ Department of Textile Engineering, Chemistry, and Science, North Carolina State University, Raleigh, NC, USA
}

\begin{abstract}
Color Index Disperse Yellow 42 (DY42), a high-volume disperse dye for polyester, was used to compare the capabilities of the LTQ-Orbitrap XL and the LTQ-FT-ICR with respect to mass measurement accuracy (MMA), spectral accuracy, and sulfur counting. The results of this research will be used in the construction of a dye database for forensic purposes; the additional spectral information will increase the confidence in the identification of unknown dyes found in fibers at crime scenes. Initial LTQ-Orbitrap XL data showed MMAs greater than 3 ppm and poor spectral accuracy. Modification of several Orbitrap installation parameters (e.g., deflector voltage) resulted in a significant improvement of the data. The LTQ-FT-ICR and LTQ-Orbitrap XL (after installation parameters were modified) exhibited MMA $\leq 3 \mathrm{ppm}$, good spectral accuracy ( $x^{2}$ values for the isotopic distribution $\leq 2$ ), and were correctly able to ascertain the number of sulfur atoms in the compound at all resolving powers investigated for AGC targets of $5.00 \times 10^{5}$ and $1.00 \times 10^{6}$.
\end{abstract}

Key words: Electrospray ionization, FT-ICR-MS, Orbitrap-MS, Forensic analysis, Textiles, Trace evidence

\section{Introduction}

$\mathrm{H}$ igh resolving power (RP) mass spectrometers have the ability to provide high mass measurement accuracy (MMA), which is invaluable in defining the elemental composition of unknowns [1-3]. However, MMA only takes into account the monoisotopic peak, not the entire isotopic distribution. High RP instruments produce spectra that contain useful isotopic information that can be utilized to provide more

Samantha L. Blake and S. Hunter Walker equally contributed to this research

Correspondence to: David C. Muddiman; e-mail: david_muddiman@ncsu. edu confident elemental compositions. Isotopic distributions contain several peaks whose relative heights are due to natural isotopic abundances and can be used to aid in the elemental composition determination of an unknown substance [4]. For example, the relative abundance of the $\mathrm{A}+1$ peak compared with the monoisotopic peak in conjunction with the natural abundance of the ${ }^{13} \mathrm{C}$ isotope is indicative of the total number of carbons in the compound [5]. The relative abundance of the $\mathrm{A}+2$ peak in the distribution can be used to accurately predict the presence and the number of atoms with $\mathrm{A}+2$ isotopes, such as sulfur, bromine, and chlorine $[4,6]$.

Spectral accuracy is the ability of the mass spectrometer to correctly measure the isotopic distribution of ions [7]. Accurate isotopic ratios are necessary to increase confidence 
in elemental composition assignments. Isotopic distribution evaluation analyses have been reported in the literature using Orbitrap [8] and FT-ICR spectra [9] as a way to reduce the number of possible elemental composition assignments for a given species. Junot et al. found that absolute ion abundance was the main factor affecting the isotopic distribution and mass accuracy while studying isotopic distributions in the LTQ-Orbitrap [8]. They also found that liquid chromatography improved the relative isotopic abundances of the distribution, which is attributed to decreasing the number of isotopic distributions present [8]. Stoll et al. evaluated different methods, such as calculated double-bond equivalence, ion state, and isotopic distribution simulation, to compare theoretical and experimental data as a means of narrowing elemental composition assignments [9]. In the 25 compounds studied, they found that isotopic distribution evaluation led to a decrease in the number of possible elemental composition assignments for a given species by an average of $90.6 \%$ [9]. Thus, the accurate measurement of not only the mass (MMA), but also the isotopic distribution can be significantly exploited for the confident identification of elemental compositions in high RP mass analysis.

Using high RP MS, it is also possible to count (in some cases estimate) the number of specific atoms $(\mathrm{C}, \mathrm{S}, \mathrm{Br}, \mathrm{Cl}$, etc.) in a compound through analysis of the $\mathrm{A}+1$ and $\mathrm{A}+2$ isotopic peaks. For example, using the natural abundance of the ${ }^{34} \mathrm{~S}$ atom and the relative height of the resolved doublet at the $\mathrm{A}+2$ peak, the number of sulfurs in a molecule can be determined. The natural abundance of the ${ }^{34} \mathrm{~S}$ isotope is $3.976 \%-4.734 \%$, as reported by NIST [10]. When sulfur is present in a compound, high RP MS $(\geq 30,000)$ is capable of resolving the $\mathrm{A}+2$ peak into a doublet with a mass difference of $0.0109 \mathrm{Da}$ between the peaks. The lighter peak, indicative of the ${ }^{34} \mathrm{~S}_{1}$ isotope, has a mass difference of $1.9958 \mathrm{Da}$ from the monoisotopic mass and the heavier peak, indicative of the ${ }^{13} \mathrm{C}_{2}$ isotopic peak, has a mass difference of $2.0067 \mathrm{Da}$ from the monoisotopic mass [6]. The relative abundance of the ${ }^{34} \mathrm{~S}$ peak in comparison to the monoisotopic peak depends on the number of sulfur atoms present in the compound. For each sulfur present in the compound, the relative abundance of the ${ }^{34} \mathrm{~S}$ peak increases by the natural abundance of the ${ }^{34} \mathrm{~S}$ isotope. The ability of high RP mass spectrometers to count sulfurs has been studied previously [11-13]. Marshall and coworkers used sulfur-counting as a means to characterize the p16 tumor suppressor protein [11], and also later in the study of glycosphingolipids [13]. Hoye and coworkers applied sulfurcounting to the study of sea lamprey pheromones [12].

Automatic gain control (AGC) target is a means of controlling the ion population during analysis [14, 15]. Higher ion populations increase space charge effects and can lead to decreased MMA; additionally, variable ion populations can allow for variable MMA and spectral accuracy for each individual spectrum. Thus, AGC allows one to keep the total ion population 'constant', which significantly decreases the analytical variability of the measurement [16].
In this paper, the capabilities of the LTQ-FT-ICR and the LTQ-Orbitrap XL for determination of elemental composition through utilization of MMA, spectral accuracy, and sulfur counting are compared. AGC target and resolving power were varied in both the LTQ-Orbitrap XL and the LTQ-FT-ICR as a means of understanding the capabilities and limitations of the instrumentation pertaining to small molecule analysis. Furthermore, the authors present two separate data sets acquired on the LTQ-Orbitrap XL. The initial data set taken on the LTQ-Orbitrap XL produced spectra with MMA $>2.5 \mathrm{ppm}$ and inaccurate sulfur counting analyses despite the instrument passing all user-tuning procedures and being calibrated per the manufacturer specifications. Thus, detailed inspection and manual tuning of the installation parameters by the manufacturer followed, and sub-ppm MMA was achieved in subsequent data acquisition (vide infra).

\section{Experimental}

\section{Disperse Yellow 42 Sample Preparation}

Color Index (C.I.) Disperse Yellow 42, $\mathrm{C}_{18} \mathrm{H}_{15} \mathrm{~N}_{3} \mathrm{O}_{4} \mathrm{~S}$, (DY 42), trade name Foron Yellow AS-FL, was obtained from Clariant (Charlotte, NC), batch CHAA1097752C for analysis. To purify the dye powder for analysis, raw dye powder $(20 \mathrm{mg}$ ) was dissolved in acetone $(20 \mathrm{~mL})$ and brought to a boil while stirring on a hot plate. The sample was filtered hot, and the filtrate was evaporated to dryness. The dried sample was collected for analysis.

Two stock solutions $(1 \mathrm{mg} / \mathrm{mL})$ of the purified dye powder were prepared in 50:50 acetonitrile:water. The samples were diluted to $10 \mu \mathrm{g} / \mathrm{mL}$ for analysis on a hybrid linear ion trap, Orbitrap XL mass spectrometer, LTQOrbitrap XL (Thermo Scientific, San Jose, CA) and a hybrid linear ion trap, Fourier transform ion cyclotron resonance mass spectrometer, LTQ-FT-ICR, (Thermo Scientific) with a 7 Tesla superconducting magnet.

\section{LTQ- FT-ICR Analysis}

The instrument was calibrated per the manufacturer specifications. The $10 \mu \mathrm{g} / \mathrm{mL}$ sample was introduced by direct injection via an in-house nanoESI source. The following instrument parameters were used for all analyses on the LTQ-FT-ICR: capillary voltage and temperature of $42 \mathrm{~V}$ and $225{ }^{\circ} \mathrm{C}$, respectively, and a tube lens voltage of $120 \mathrm{~V}$. Thirty full range mass spectra $(150-2000 \mathrm{~m} / \mathrm{z})$ were collected at two AGC targets: $5.00 \times 10^{5}$ and $1.00 \times 10^{6}$. The full-width-half-maximum resolving powers $\left(\mathrm{RP}_{\mathrm{FWHM}}\right.$ at $\left.m / z=400\right)$ evaluated were $12,500,25,000,50,000,100,000$, and 200,000.

\section{LTQ-Orbitrap XL Analysis}

The instrument was calibrated per the manufacturer specifications with the lock-mass feature enabled. All instrument 
parameters were the same as specified for the LTQ-FT-ICR (vide supra). The resolving power and AGC target were varied and 30 full range $(200-2000 \mathrm{~m} / \mathrm{z})$ spectra were collected at each set of conditions. The following $\mathrm{RP}_{\mathrm{FWHM}}$ at $m / z=400$ were used during the LTQ-Orbitrap XL analysis: 7,500, 15,000, $30,000,60,000$, and 100,000 at $m / z=400$. The same AGC targets were used as with the LTQ-FT analysis.

\section{Data Analysis}

The MMA, $\chi^{2}$ value for the isotopic distribution, and relative abundance of the $\mathrm{A}+2$ sulfur peak were calculated. Equation (1) is the formula used to calculate the MMA of the monoisotopic peak at each resolving power and AGC target.

$$
\operatorname{MMA}(\mathrm{ppm})=\frac{\text { Experimental }- \text { Theoretical }}{\text { Theoretical }} \times 10^{6}
$$

The $\chi^{2}$ value for the relative abundances in relation to the monoisotopic peak in the isotopic distribution of DY 42 was calculated to quantify spectral accuracy; shown as equation (2) [17]. The $\chi^{2}$ value was calculated for each spectrum at each resolving power and AGC target studied.

$$
\mathrm{x}^{2}=\sum \frac{(\text { Observed }- \text { Expected })^{2}}{\text { Expected }}
$$

The number of sulfurs was found by determining the relative abundance of the sulfur peak compared to the monoisotopic peak and multiplying by the ${ }^{32} \mathrm{~S}:{ }^{34} \mathrm{~S}$ isotope natural composition ratio where $\mathrm{A}+2$ and $\mathrm{A}$ are the experimental peak intensities of the ${ }^{34} \mathrm{~S}$ and monoisotopic peak, respectively. ${ }^{32} \mathrm{~S}_{\text {Theoretical }}$ and ${ }^{34} \mathrm{~S}_{\text {Theoretical }}$ are the natural isotopic compositions as reported by NIST of the ${ }^{32} \mathrm{~S}$ and ${ }^{34} \mathrm{~S}$, respectively

$$
\frac{\mathrm{A}+22_{\text {Experimental }}}{\mathrm{A}_{\text {Experimental }}} \times \frac{{ }^{32} \mathrm{~S}_{\text {Theoritical }}}{{ }^{34} \mathrm{~S}_{\text {Theoritical }}}=\# \text { of Sulfurs }
$$

\section{Results and Discussion}

Two data sets will be discussed for the LTQ-Orbitrap XL in comparison to a data set produced by the LTQ-FT-ICR. The initial data set for the LTQ-Orbitrap XL produced results that are not consistent with the specifications reported for an Orbitrap instrument by the manufacturer. Additionally, despite passing all user tuning methods and being calibrated per the manufacturer specifications, this group has observed sub-par MMA for this instrument since installation. Furthermore, before the installation parameters were adjusted, sulfur counting experiments in the LTQ-Orbitrap XL did not produce the correct number of sulfurs for a standard molecule, DY42. Thus, in a personal communication with
Alexander Makarov, he immediately concluded that the Orbitrap was not performing optimally upon assessment of the data. During a remote session on the instrument, specific installation parameters of the instrument were manually tuned. These installation parameters are not included in the user tune method, and it is not recommended that these parameters be changed by anyone other than a service engineer. The parameters that were changed include deflector voltage on the Orbitrap and the injection level of the central electrode along
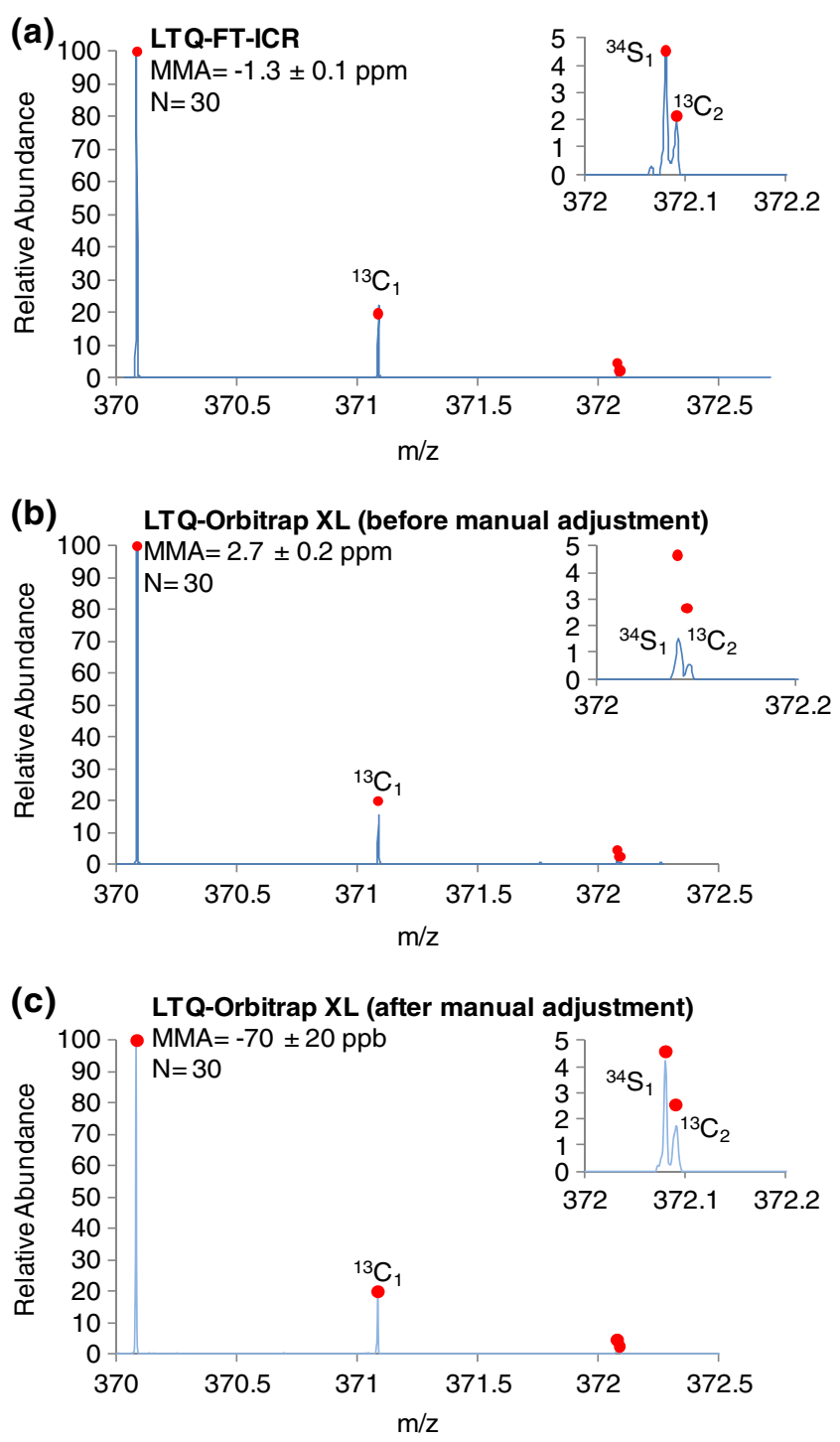

Figure 1. Single acquisition FTMS spectra of disperse yellow 42 dye: (a) spectrum acquired on 7 Tesla LTQ-FT-ICR mass spectrometer at AGC $1.00 \times 10^{6}$ and $\mathrm{RP}_{\mathrm{FWHM}} 100,000$ at $\mathrm{m} / \mathrm{z}=$ 400, (b) spectrum acquired on LTQ-Orbitrap XL at AGC of $1.00 \times 10^{6}$ and $\operatorname{RP}_{\mathrm{FWHM}} 60,000$ at $m / z=400$ before manual adjustment, and (c) spectrum acquired on the LTQ-Orbitrap $\mathrm{XL}$ at $\mathrm{AGC}$ of $1.00 \times 10^{6}$ and $\mathrm{RP}_{\mathrm{FWHM}} 60,000$ at $\mathrm{m} / \mathrm{z}=400$ after manual adjustment. The red dots indicate the relative abundances of the theoretical isotope distribution. The inset shows the $A+2$ peak demonstrating that the ability of both instruments to resolve the ${ }^{34} S_{1}$ peak from the ${ }^{13} C_{2}$ peak 
with minor adjustments to the lens voltages and pulse voltages on the C-trap. Upon manual optimization of these installation parameters, the second set of data from the LTQ-Orbitrap XL was collected, which produced significantly higher quality data with respect to MMA and spectral accuracy.

Figure 1a, b, and c are example spectra of each data set for DY 42 produced by LTQ-FT-ICR, LTQ-Orbitrap XL (before manual adjustment of the installation parameters), and LTQOrbitrap XL (after manual adjustment of the installation parameters), respectively. The LTQ-FT-ICR spectra were taken at a $\mathrm{RP}_{\mathrm{FWHM}}$ at $m / z=400$ of 100,000 and an AGC target of $1.00 \times 10^{6}$ Both the LTQ-Orbitrap XL spectra in Figure $1 \mathrm{~b}$ and c (before and after manual adjustment of the installation parameters, respectively) were acquired at an $\mathrm{RP}_{\mathrm{FWHM}}$ at $m / z=400$ of 60,000 and an AGC target of $1.00 \times 10^{6}$. The red dots above each peak in all three spectra are indicative of the theoretical peak heights for the isotopic distribution of DY 42 . Figure $1 \mathrm{~b}$ and $\mathrm{c}$ display the vast difference in the results acquired before and after manual adjustment of the installation parameters. The MMA is an order of magnitude better, and the spectral accuracy is significantly better (lower $\chi^{2}$ value - vide infra) upon adjustment of the installation parameters. These data suggest that a standard molecule or procedure must be used (in addition to the tuning protocol provided by the manufacturer) to determine whether or not the instrument is performing optimally. Importantly, upon manual adjustment of the LTQ-Orbitrap
XL installation parameters, Figure 1a (LTQ-FT-ICR) and Figure 1c (LTQ-Orbitrap XL) show that the two instrument platforms are capable of acquiring the correct results.

\section{Mass Measurement Accuracy}

Plots of the MMA of the monoisotopic peak of each spectrum and the average MMA with $95 \%$ confidence intervals at each resolving power are shown for the LTQFT-ICR and LTQ-Orbitrap XL (after installation parameter adjustment) in Figure 2. Figure 2a and $\mathrm{c}$ are shown at an AGC target of $5.00 \times 10^{5}$ and Figure $2 \mathrm{~b}$ and $\mathrm{d}$ are shown at an AGC target of $1.00 \times 0^{6}$. The oleamide ion $(\mathrm{m} / \mathrm{z} 282.2791)$ was used for lock-mass during the LTQ-Orbitrap XL analysis. Lock-mass is an internal $\mathrm{m} / \mathrm{z}$ standard used to correct for fluctuations due to temperature and high voltages $[1,18]$ and can allow for sub-ppm MMA.

Spectra acquired on the LTQ-FT-ICR produced MMA below $2 \mathrm{ppm}$ for AGC targets $5.00 \times 10^{5}$ and $1.00 \times 10^{6}$. The average $\mathrm{MMA} \pm 95 \%$ confidence interval of 30 spectra produced by the LTQ-FT-ICR at AGC target $1.00 \times 10^{6}$ and $\mathrm{RP}_{\mathrm{FWHM}} 100,000$ was $-1.3 \pm 0.1 \mathrm{ppm}$ (Figure 1a). The average $\mathrm{MMA} \pm 95 \%$ confidence interval of 30 spectra produced on the LTQ-Orbitrap XL, before adjustment of the installation parameters, at AGC target $1.00 \times 10^{6}$ and $\mathrm{RP}_{\mathrm{FWHM}} 60,000$ was $2.7 \pm 0.2 \mathrm{ppm}$ (Figure 1b). After the installation parameter
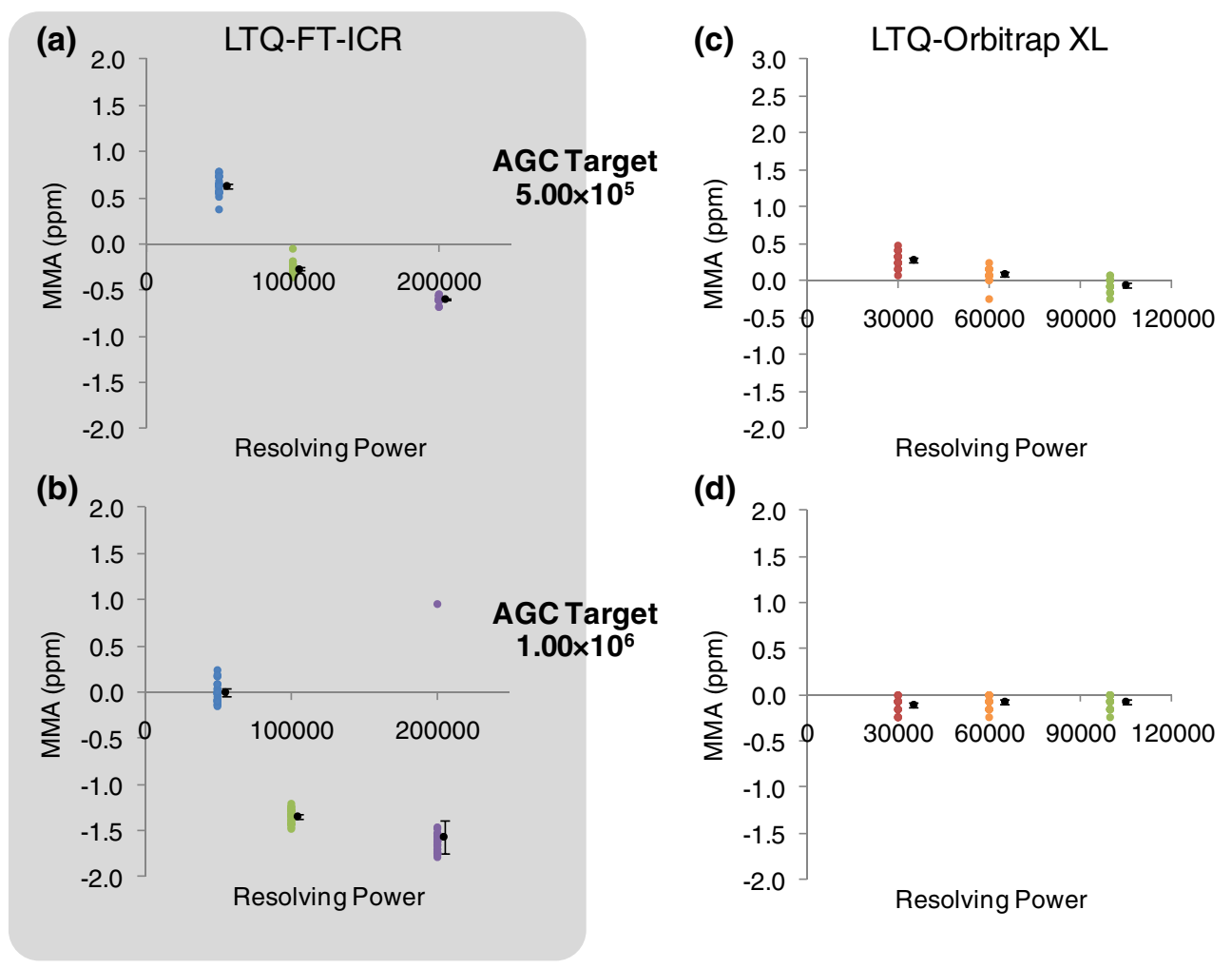

Figure 2. The calculated MMA of the monoisotopic peak for the LTQ-FT-ICR data (a) and (b) and the LTQ-Orbitrap XL data after manual adjustment (c) and (d) are plotted against the $\mathrm{RP}_{\mathrm{FWHM}}$ at $m / z=400$. The series in black indicates the averages and the $95 \%$ confidence intervals of the measurement $(\mathrm{N}=30)$. The data points next to each averaged point are the calculated MMAs for each individual spectrum acquired. The AGC target values evaluated: (a) and (c) $5.00 \times 10^{5}$ and (b) and (d) $1.00 \times 10^{6}$ 
optimization on the LTQ-Orbitrap XL, the average MMA \pm $95 \%$ confidence interval of 30 spectra at AGC target $1.00 \times 10^{6}$ and $\mathrm{RP}_{\mathrm{FWHM}} 60,000$ was $-70 \pm 20$ ppb (Figure 1c). Upon correction of the LTQ-Orbitrap XL, both instruments performed within manufacturers specifications; however, with lock-mass enabled, the LTQ-Orbitrap XL is capable of ppb mass accuracy. A consistent positive bias and MMA values routinely greater than $2.5 \mathrm{ppm}$ were observed in the LTQOrbitrap XL data before parameter optimization (data not shown) at all resolving powers for AGC targets $5.00 \times 10^{5}$ and $1.00 \times 10^{6}$. After the parameters mentioned above were adjusted on the LTQ-Orbitrap XL, the data quality improved significantly with MMA values routinely less than $1 \mathrm{ppm}$.

\section{Spectral Accuracy and Sulfur Counting}

The $\chi^{2}$ value of the isotopic distribution was used to "quantify" spectral accuracy in these experiments; a high $\chi^{2}$ value is indicative of poor spectral accuracy. The calculated $\chi^{2}$ values as a function of resolving power can be seen in Figure 3 at AGC targets of $5.00 \times 10^{5}$ (Figure $3 \mathrm{a}$ and c) and $1.00 \times 10^{6}$ (Figure $3 \mathrm{~b}$ and $d$ ), for both the LTQ-FT-ICR (Figure $3 a$ and $b$ ) and the LTQ-Orbitrap XL (Figure 3c and d). The sulfur peak relative abundance can be seen on the left y-axis of the same plots.
Spectral accuracy plots for initial data produced by the LTQOrbitrap XL before manual adjustment (data not shown) suggest a decrease in spectral accuracy as the resolving power increased, agreeing with the findings of Erve et al. [7]; however, this correlation was not observed after the instrument parameters were changed on the Orbitrap or in any of the data collected on the LTQ-FT-ICR. After optimization of the mentioned installation parameters, spectral accuracy in the LTQ-Orbitrap XL improved by $84.4 \%$.

Erve et al. noted that spectral accuracy decreased as resolving power increased; they attributed this to isotopic beating $[7,19,20]$. The closer two frequencies are the longer the beat period meaning the specific isotopes interact less during the measurement period. However, two species with similar frequencies can also undergo peak coalescence. When deriving conclusions, one must consider the isotopic beat period, measurement period, and window function applied as this will have an effect on the isotopic distributions. Moreover, in their work, the isotopic fine structure was unresolved, in large part due to the fact the compounds they studied had a significantly higher $\mathrm{m} / \mathrm{z}$ (lower frequency) than this study. In this study, we were able to resolve the ${ }^{13} \mathrm{C}_{2}$ from the ${ }^{34} \mathrm{~S}_{1}$ isotopic peaks and, thus, determine their individual frequencies in both the LTQ-
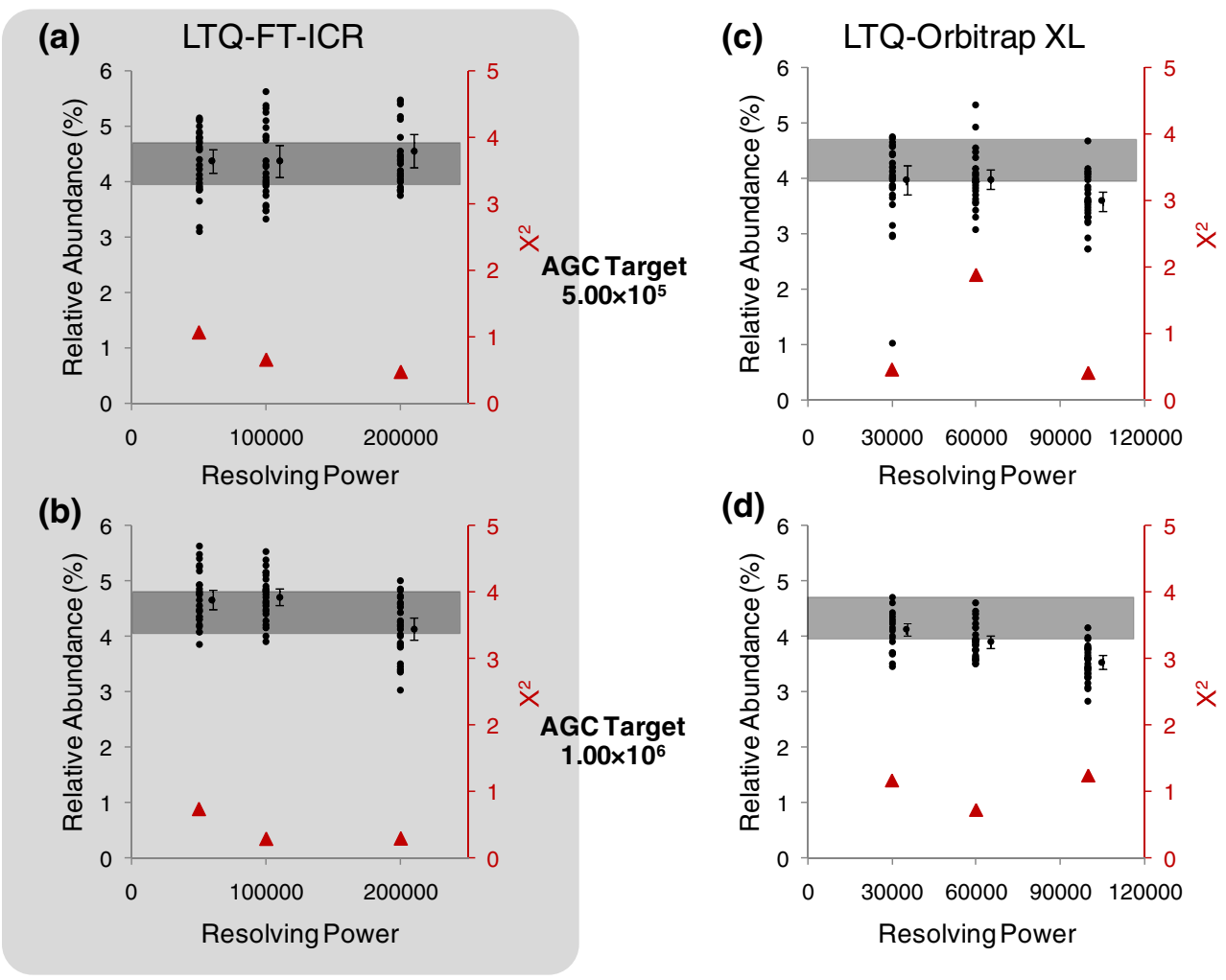

Figure 3. The relative abundance of ${ }^{34} \mathrm{~S}_{1}$ to the monoisotopic peak (left hand axis) for the LTQ-FT-ICR data (a) and (b) and the LTQOrbitrap XL data after manual adjustment (c) and (d) are plotted against the $\mathrm{RP}_{\mathrm{FWHM}}$ at $m / z=400$. The $\mathrm{X}^{2}$ value for the entire isotopic distribution is also plotted (right hand axis) with the red filled triangles ( $\mathbf{\Delta})$ being the calculated $x^{2}$ value. The average sulfur peak abundance $(\mathrm{N}=30)$ is the black circle series with $95 \%$ confidence interval error bars. The points next to each averaged point are the sulfur peak abundances for each individual spectrum. The gray bar indicates the range of natural variation of the ${ }^{34} \mathrm{~S}$ isotope as reported by NIST, 3.976\%-4.734\% [10]. The AGC target values evaluated: (a) and (c) $5.00 \times 10^{5}$ and (b) and (d) $1.00 \times 10^{6}$ 
FT-ICR and the LTQ Orbitrap XL using the diagnostic mode. The isotopic beat periods for this doublet were 0.116 and $0.196 \mathrm{~s}$ in the LTQ-FT-ICR and LTQ-Orbitrap XL, respectively, which is small relative to the measurement period $(\sim 1 \mathrm{~s})$ for a typical analysis. In contrast, the beat period for the monoisotopic peak and the $\mathrm{A}+1$ peak in the LTQ-FT-ICR and LTQ-Orbitrap XL were $\sim 0.0013$ and $\sim 0.002 \mathrm{~s}$, respectively. While the beat period is much longer for the $\mathrm{A}+2$ doublets relative to the ${ }^{13} \mathrm{C}$ isotopes (a factor of $\sim 90$ for the LTQ-FT-ICR and 149 for the LTQ-Orbitrap) this should be sufficient to provide high spectral accuracy for the entire isotopic distribution. This is consistent with our findings. We are currently pursuing these incongruent observations, keeping in mind these are different molecular systems studies, with more detailed studies.

The sulfur peak relative abundances produced by the LTQ-FT-ICR were within the expected natural abundance range for one sulfur atom at all resolving powers studied for AGC targets $5.00 \times 10^{5}$ and $1.00 \times 10^{6}$, demonstrating the capability of the LTQ-FT-ICR to accurately resolve the ${ }^{34} \mathrm{~S}_{1}$ and ${ }^{13} \mathrm{C}_{2}$ peaks and quantify the number of sulfurs.

Analysis of the $\mathrm{A}+2$ peak of the spectrum for the LTQOrbitrap XL data acquired before manual adjustment showed peak heights $(\leq 3 \%$ relative abundance) systematically lower than the theoretical abundance, which did not give an accurate indication of the number of sulfur atoms in the compound (data not shown); however, the $\mathrm{A}+2$ peak heights for the data taken after the LTQ-Orbitrap XL installation parameters were changed are within the expected peak height range for a compound containing one sulfur. The average sulfur peak height $\pm 95 \%$ confidence interval for the LTQ-Orbitrap XL at AGC target $1.00 \times 10^{6}$ and $\mathrm{RP}_{\mathrm{FWHM}}$ 60,000 before the parameter optimization was $1.82 \pm 0.11$, compared with $3.91 \pm 0.21$ after parameter optimization.

\section{Conclusions}

The small molecule analysis capabilities in the context of MMA, spectral accuracy, and sulfur counting were compared for the LTQ-FT-ICR and the LTQ-Orbitrap XL. Both instruments were reported by Thermo Fisher Scientific to be performing at manufacturer specifications, but initially the LTQ-Orbitrap XL was far outperformed by the LTQ-FTICR. A more in-depth inspection of the LTQ-Orbitrap XL installation parameters, outside of the user tuning procedure, yielded significantly improved data. Sulfur counting was successful using the LTQ-FT-ICR and LTQ-Orbitrap XL platforms at AGC targets $5.00 \times 10^{5}$ and $1.00 \times 10^{6}$. Based on these results, the LTQ-Orbitrap XL is a suitable replacement technology for the LTQ-FT-ICR for small molecule sulfur counting and isotopic distribution analysis to yield more confident elemental composition assignments.

Due to the LTQ-Orbitrap XL passing all manufacturer tuning procedures, yet still acquiring data with $\geq 2.5 \mathrm{ppm}$ MMA in the experiments before the manual adjustment, the authors suggest that further inspection may be needed to manually and regularly asses the performance of the LTQOrbitrap XL. A simple assessment of performance is spectral accuracy analysis of the LTQ-Orbitrap calibration mix. This mix includes MRFA (Met-Arg-Phe-Ala peptide, $\mathrm{m} / z$ 524), which contains one sulfur atom. Thus, the height of the resolved ${ }^{34} \mathrm{~S}$ peak in the $\mathrm{A}+2$ peak of the normalized MRFA isotopic distribution should be within the natural abundance levels of ${ }^{34} \mathrm{~S}$ and can be used to determine if the instrument is out of specification and whether adjustment of the parameters mentioned needs to be considered. Specifically, the average relative intensity of the $\mathrm{A}+2$ peak measured in SIM or $\mathrm{MS}^{2}$ mode (centered on $\mathrm{m} / \mathrm{z} 524$ with isolation width of at least $10 \mathrm{Da}, \mathrm{AGC}$ target $1.00 \times 10^{5}$ in either mode and 0 collision energy, first $m / z 150$ for MS2 mode) should not be lower than 3\% for resolving power settings 60,000 and 100,000 [18] (preferably, it should be around 4\%). This will allow for facile and routine assessment of the LTQ-Orbitrap $\mathrm{XL}$ performance in order to assure the highest possible quality of data is acquired.

\section{Acknowledgments}

The authors thank Dr. Alexander Makarov and Dr. Eduard Denisov for their assistance in diagnosing and correcting LTQ-Orbitrap XL installation parameters outside the scope of the available user tuning and calibration procedures, Ms. Kristin Cochran for her assistance with data work-up, and Dr. D. Keith Williams for his helpful suggestions. The authors acknowledge generous support from the National Science Foundation (IIS0941421), the NIH/NCSU Molecular Biotechnology Training Program [5T32GM00-8776-08, (S.H.W.)], the W. M. Keck Foundation, and North Carolina State University.

\section{References}

1. Olsen, J.V., de Godoy, L.M.F., Li, G.Q., Macek, B., Mortensen, P., Pesch, R., Makarov, A., Lange, O., Horning, S., Mann, M.: Parts per million mass accuracy on an orbitrap mass spectrometer via lock mass injection into a C-trap. Mol. Cell. Proteom. 4, 2010-2021 (2005)

2. Han, J., Danell, R.M., Patel, J.R., Gumerov, D.R., Scarlett, C.O., Speir, J.P., Parker, C.E., Rusyn, I., Zeisel, S., Borchers, C.H.: Towards highthroughput metabolomics using ultrahigh-field fourier transform ion cyclotron resonance mass spectrometry. Metabolomics 4, 128-140 (2008)

3. Makarov, A., Denisov, E., Lange, O., Horning, S.: Dynamic range of mass accuracy in LTQ orbitrap hybrid mass spectrometer. J. Am. Soc. Mass Spectrom. 17, 977-982 (2006)

4. Kind, T.; Fiehn, O. Metabolomic Database Annotations Via Query of Elemental Compositions: Mass Accuracy is Insufficient Even at Less than 1 ppm. BMC Bioinformatics 7, 234 (2006)

5. Watson, J.T., Sparkman, O.D.: Introduction to Mass Spectrometry, 4th edn. John Wiley and Sons, Ltd, West Sussex (2007)

6. Grange, A.H., Donnelly, J.R., Sovocool, G.W., Brumley, W.C.: Determination of elemental compositions from mass peak profiles of the molecular ion (M) and the $\mathrm{M}+1$ and $\mathrm{M}+2$ ions. Anal. Chem. 68, 553-560 (1996)

7. Erve, J.C.L., Gu, M., Wang, Y.D., DeMaio, W., Talaat, R.E.: Spectral accuracy of molecular ions in an LTQ/Orbitrap mass spectrometer and implications for elemental composition determination. J. Am. Soc. Mass Spectrom. 20, 2058-2069 (2009)

8. Xu, Y., Heilier, J.F., Madalinski, G., Genin, E., Ezan, E., Tabet, J.C., Junot, C.: Evaluation of accurate mass and relative isotopic abundance 
measurements in the LTQ-Orbitrap mass spectrometer for further metabolomics database building. Anal. Chem. 82, 5490-5501 (2010)

9. Stoll, N., Schmidt, E., Thurow, K.: Isotope pattern evaluation for the reduction of elemental compositions assigned to high-resolution mass spectral data from electrospray ionization fourier transform ion cyclotron resonance mass spectrometry. J. Am. Soc. Mass Spectrom. 17, 1692-1699 (2006)

10. Bohlke, J.K., de Laeter, J.R., De Bievre, P., Hidaka, H., Peiser, H.S., Rosman, K.J.R., Taylor, P.D.P.: Isotopic compositions of the elements, 2001. J. Chem. Phys. Ref. Data 34, 57-67 (2005)

11. Shi, S.D.H., Hendrickson, C.L., Marshall, A.G.: Counting individual sulfur atoms in a protein by ultrahigh-resolution fourier transform ion cyclotron resonance mass spectrometry: experimental resolution of isotopic fine structure in proteins. Proc. Natl. Acad. Sci. U.S.A. 95, 11532-11537 (1998)

12. Hoye, T.R., Dvornikovs, V., Fine, J.M., Anderson, K.R., Jeffrey, C.S., Muddiman, D.C., Shao, F., Sorensen, P.W., Wang, J.: Details of the structure determination of the sulfated steroids PSDS and PADS: new components of the Sea Lamprey (Petromyzon marinus) migratory pheromone. J. Org. Chem. 72, 7544-7550 (2007)

13. He, H., Emmett, M.R., Nilsson, C.L., Conrad, C.A., Marshall, A.G.: High mass accuracy and resolution facilitate identification of glycosphingolipids and phospholipids. Int. J. Mass Spectrom. 305, 116-119 (2011)

14. Belov, M.E., Zhang, R., Strittmatter, E.F., Prior, D.C., Tang, K., Smith, R.D.: Automated gain control and internal calibration with external ion accumulation capillary liquid chromatography-electrospray ionizationfourier transform ion cyclotron resonance. Anal. Chem. 75, 4195-4205 (2003)
15. Syka, J.E.P., Marto, J.A., Bai, D.L., Horning, S , Senko, M.W., Schwartz, J.C., Ueberheide, B., Garcia, B., Busby, S., Muratore, T., Shabanowitz, J., Hunt, D.F.: Novel linear quadrupole ion Trap/FT mass spectrometer: performance characterization and use in the comparative analysis of histone $\mathrm{H} 3$ post-translational modifications. J. Proteome Res. 3, 621-626 (2004)

16. Williams, D.K., Muddiman, D.C.: Parts-per-billion mass measurement accuracy achieved through the combination of multiple linear regression and automatic gain control in a fourier transform ion cyclotron resonance mass spectrometer. Anal. Chem. 79, 5058-5063 (2007)

17. Senko, M.W., Beu, S.C., Mclafferty, F.W.: Determination of monoisotopic masses and ion populations for large biomolecules from resolved isotopic distributions. J. Am. Soc. Mass Spectrom. 6, 229233 (1995)

18. Perry, R.H., Cooks, R.G., Noll, R.J.: Orbitrap mass spectrometry: instrumentation, ion motion, and applications. Mass Spectrom. Rev. 27, 661-699 (2008)

19. Hofstadler, S.A., Bruce, J.E., Rockwood, A.L., Anderson, G.A., Winger, B.E., Smith, R.D.: Isotopic beat patterns in fourier transform ion cyclotron resonance mass spectrometry: implications for high resolution mass measurements of large biopolymers. Int. J. Mass Spectrom. Ion Processes 132, 109-127 (1994)

20. Gordon, E.F., Muddiman, D.C.: Impact of ion cloud densities on the measurement of relative ion abundances in fourier transform ion cyclotron resonance mass spectrometry: experimental observations of coulombically induced cyclotron radius perturbations and ion cloud dephasing rates. J. Mass Spectrom. 36, 195-203 (2001) 